\title{
Temas y pretextos para descubrir una región: El patrimonio gastronómico y sus potencialidades como objeto de estudio y enseñanza ${ }^{1}$
}

Fabián Andrés Llano ${ }^{2}$

\section{Resumen}

El siguiente artículo de revisión tiene como propósito evidenciar las tendencias y los enfoques teóricos y metodológicos del patrimonio gastronómico como objeto de estudio. Además de las problemáticas asociadas a este objeto en particular, las diferentes líneas de pensamiento halladas en esta revisión documental permitieron abrir posibilidades de pensar el patrimonio gastronómico desde el trabajo interdisciplinar y sus potencialidades para el desarrollo económico sostenible. Desde esta última perspectiva, aparecen formas de abordaje desde la enseñanza y la formación de futuros profesionales de la gastronomía por medio del respeto a las tradiciones locales y la puesta en valor de los territorios y los productos de la tierra.

Palabras claves: patrimonio gastronómico, alimento, costumbres y tradiciones, desarrollo económico y social, turismo alimentario.

\section{Introducción}

El patrimonio gastronómico hoy día se ha convertido en un asunto de gran interés para la comunidad científica y las diferentes agencias encargadas del desarrollo económico. Las potencialidades que posee para convertirse en un insumo importante del desarrollo económico, han convertido al patrimonio gastronómico en un asunto de suma importancia en los planes de desarrollo. Como factor potencial del turismo, del desarrollo territorial y por su

\footnotetext{
${ }^{1} \mathrm{El}$ siguiente texto hace parte de una estrategia idea por la Universidad Uniagustiniana para el fortalecimiento de las líneas de investigación de sus programas. En este caso específico, la elaboración de un estado del arte relacionado con un área estratégica asociada al patrimonio cultural y gastronómico.

2 Doctor en Ciencias Humanas del Patrimonio y la Cultura Universidad de Girona (España) Magister en Investigación social interdisciplinaria de la Universidad Distrital Francisco José de Caldas, Licenciado en Ciencias Sociales. En la actualidad se desempeña como docente investigador del programa Administración Turística y Hotelera de la universitaria Uniagustiniana (Bogotá-Colombia) ID 0000-0003-2181-3476
} 
puesto de la actividad económica y científica, el patrimonio gastronómico se postula como uno de los principales activos económicos y culturales de un país (Pérez Sánchez M., Cisneros Mújica W, 2006, Llano, 2013, Clemente E., Hernández J. M., López-Guzmán T. 2014, Suremain, C, 2017, Medina, 2017). Esta apertura hacia el reconocimiento de las diferentes tradiciones y sus acervos alimentarios permite entrever un filón de oportunidades que tiene el patrimonio gastronómico como elemento indispensable para la articulación de la actividad económica con la cultura (Torres G., Madrid de Zito F. L. y Santoni M. (s.f.), Pérez Sánchez M., Cisneros Mújica W, 2006; Lourdes, A. 2006, Tomás, L., y Margarida, JM 2011). Ya sea como actividad científica o como elemento económico, el patrimonio gastronómico apunta cada vez más a ser tenido en cuenta en las principales agendas de los gobiernos para respaldar un desarrollo económico sostenible (Espeitx, E. 2004).

Como uno de los puntos centrales de las agendas públicas en diferentes regiones, el tema del patrimonio gastronómico requiere ser comprendido desde un ángulo mucho más relacionado con el desarrollo científico bajo su abordaje como objeto de estudio. Para lograr descifrar las potencialidades del patrimonio gastronómico para el desarrollo de las regiones y el impacto de su reconocimiento en los diferentes territorios, se planteó la necesidad de un rastreo de los principales estudios del patrimonio gastronómico en relación con diferentes elementos culturales y territoriales. Desde este panorama inicial se hizo indispensable plantearse la pregunta por las tendencias, los enfoques o los paradigmas donde se inscribe la conceptualización del patrimonio gastronómico como objeto de estudio.

Estos cuestionamientos iniciales fueron abordados desde unos intereses particulares, como los referidos a la relación del alimento con los procesos de patrimonialización, que cabe advertir se han inscrito dentro de un campo mayor como es el de la cultura (Yudice, G. 2002, Fusté-Forné, F. 2016). De este modo, la pregunta por el patrimonio gastronómico tuvo como principal propósito develar los procesos de legitimación del alimento, como también sus relaciones con el territorio y los procesos de representación con los cuales se logran estructurar procesos de identidad cultural ${ }^{3}$.

\footnotetext{
${ }^{3}$ De acuerdo con Llano, (2015) la pregunta por el patrimonio gastronómico como objeto de estudio, estaría dirigida a poder a preguntarse por la legitimidad de los alimentos y los contextos de su producción, además
} 
De acuerdo con Fernández G., Agrelo E. (2003) estas relaciones del patrimonio gastronómico con los procesos de identidad no solo muestran los intercambios simbólicos, sino que además proyectan su relación casi directa con la ciudad, en la medida que la producción de alimentos y su circulación se basa en la relación recíproca entre lo rural y lo urbano. Bajo estas relaciones simbólicas e identitarias se hizo relevante hacerse las siguientes preguntas: ¿cuál es la legitimidad de los alimentos? ¿cuáles son los contextos de su producción? ¿cuál es la relación de los alimentos con la estructuración de una identidad cultural? ¿cuál es su potencial para representar a quienes consumen determinados alimentos? De acuerdo con Uribe Merino J. F. (2006) estas preguntas y otras más se relacionan con asuntos de alta envergadura como por ejemplo el problema de la seguridad alimentaria y sobre todo con el tema de la soberanía alimentaria. Otras preguntas derivadas de estas primeras reflexiones apuntan a develar algunas respuestas frente a la pregunta por el plato nacional, el papel fundamental de regiones alimentarias que rebasan la idea de organización político administrativa del territorio y por supuesto el problema cada vez más agudo de las prácticas culturales asociadas a las formas de consumo del alimento.

Más allá de la pregunta obligada sobre el plato nacional y las múltiples formas que adquiere la simbolización del alimento, se hizo relevante tener en cuenta que la cultura alimentaria, las tradiciones culinarias, las narrativas y los diferentes discursos reivindican la relación entre cocina, territorio e identidad. Todos estos temas y pretextos para abordar la relación del patrimonio gastronómico con el desarrollo regional y sobre todo con el empoderamiento de poblaciones y diferentes tradiciones culturales, permiten entrever además de las resistencias culturales y los problemas contemporáneos, todas estas dinámicas de legitimación de la alimentación. Vale la pena anotar que en estos circuitos culturales ocurren ciertas transformaciones como la proliferación de alimentos procesados junto con comida típica en diferentes presentaciones modernas (tamal y lechona enlatados) y una serie de productos tradicionales procesados con formas publicitarias de presentación que no dejan sino una serie de contradicciones alimentarias.

interrogarse por las formas de estructuración de la identidad y sobre todo por su potencial para representar a quienes consumen determinados alimentos 
Estos interrogantes que pueden convertirse en insumo relevante para nuevas investigaciones, permiten además comprender que el problema del patrimonio gastronómico no está únicamente en la denominación de ciertos productos en relación a su tradición cultural (Nieva, V. L. 2006). Este tema complejo de abordar, requiere con urgencia consolidar un campo de estudio abierto y dinámico bajo procesos de trabajo interdisciplinar y trabajo colaborativo, no solo para resguardar el producto y encapsularlo en su tradición para ser expuesto como una pieza museable, sino para encontrar soluciones a problemas mayores como el hambre, la seguridad alimentaria y la pobreza. Todos estos elementos están provistos dentro de los denominados Objetivos de Desarrollo Sostenible (ODS) propuestos por Naciones Unidas, que además permiten ampliar el espectro de acción del estudio sobre el patrimonio gastronómico.

\section{El comienzo de la indagación: el patrimonio gastronómico como objeto de estudio}

Ante esta complejidad que denota el patrimonio gastronómico, se hizo conveniente para adelantar una indagación más centrada en las intencionalidades investigativas, una conceptualización inicial que permitiera delimitar esta búsqueda. Dentro de las definiciones provistas desde el área estratégica del grupo de investigación denominado Grupo integrado de investigación en Ciencias Culinarias y gastronómicas de la Universitaria Uniagustiniana, el patrimonio gastronómico está en una relación directa con las dinámicas culturales y con las posibilidades del turismo cultural. En sintonía con Toselli, C. (2006), lo que se denomina patrimonio gastronómico es producto de la cultura y de la historia al asociarse a las dinámicas de la comunidad rural, a la soberanía alimentaria construida en el tiempo y a la tradición campesina. Ahora bien, en las discusiones actuales sobre la connotación del alimento, que por mucho tiempo estuvieron únicamente soportadas en necesidades biológicas, se ha presentado una apertura que desborda el campo antropológico donde por mucho tiempo se ha legitimado la conceptualización de las prácticas y la cultura alimentaria. Estos sentidos y asunciones de la comida referidas a connotaciones biológicas y culturales hoy día presentan un espectro mucho más amplio en relación a su abordaje como objeto de estudio. Esto 
permite involucrar a otras disciplinas y saberes en la conceptualización y abordaje de problemáticas asociadas al alimento o la comida (Varios autores, 2002)

De esta manera, las categorías de cultura alimentaria, diversidad gastronómica, globalización, globalización, legitimidad gastronómica, soberanía alimentaria, Denominaciones de Origen, paisaje cultural, entre otras, hacen parte de un acervo investigativo mucho más ligado a las epistemologías de las ciencias sociales que a su mismo desarrollo desde la química, la física y la producción de alimentos asociadas las más de las veces a las ingenierías de alimentos (Langreo N. A. 2005). Desde una tensión entre las concepciones del patrimonio gastronómico y el avance tecnológico, aparecen de fondo dos tradiciones científicas que reclaman ahora la potestad de asumir este objeto de estudio. Esta tensión cada vez más visible en las discusiones académicas y científicas, permite dejar entre ver, por una parte, la tendencia al cuidado y las formas de poner en valor los referentes identitarios de los alimentos y de otro, los desarrollos científicos en las propiedades químicas, físicas y sus ventajas como productos alimenticios. En este sentido, los abordajes culturales, frente a los tecnológicos y científicos por lo general, rehúyen el trabajo colaborativo y las dinámicas de intercambio de conocimiento al dejar de manifiesto un interés particular sobre el dominio que se tiene del objeto de estudio.

Ahora bien, como objeto de estudio desde el campo científico, el patrimonio gastronómico se hace cada vez más atractivo para el trabajo interdisciplinar. Frente a su potencial de ser pensado desde diferentes representaciones y modos de objetivación, el patrimonio gastronómico como objeto de estudio, tiene grandes posibilidades de ser estudiado más allá de las agencias culturales y el trabajo de valoración interpuesto por algunos folcloristas para ser abordado ahora por diversos agentes de las ciencias sociales y naturales con una clara mediación. Esta intervención se puede realizar desde grupos de investigación interdisciplinares, trabajos colaborativos entre las epistemologías de las ciencias naturales y las epistemologías de las ciencias sociales y sobre todo desde los potenciales aportes de disciplinas, saberes o incluso paradigmas a su construcción como campo de estudio. 


\section{Metodología: Estrategia ideada para la construcción del estado del arte}

El siguiente apartado se centra en los planteamientos procedimentales para la construcción de un estado del arte para el área de investigación sobre patrimonio y cultura gastronómica. Desde esta área estratégica de la universitaria Uniagustiniana se inició una indagación sistemática del patrimonio gastronómico como objeto de estudio. Si bien estos ejercicios de conceptualización aportan a la línea de investigación disciplinar específicamente en el área de gastronomía, este documento presente convertirse en un insumo relevante para dar soporte epistemológico, teórico, metodológico y curricular de los posibles derroteros a seguir en la investigación sobre el patrimonio gastronómico colombiano.

Si bien esta indagación arrancó desde unos intereses institucionales específicos marcados por el documento denominado Área de investigación "Patrimonio y cultura Gastronómica”, fue importante trazar unos ejes conceptuales para tal búsqueda. De esta manera, el planteamiento de una metodología para la elaboración de este estado del arte procedió bajo un ejercicio sistemático que incluyó varias consideraciones a saber: en primer lugar, que la construcción de un estado del arte obedece a unos intereses investigativos particulares y en ocasiones a unas intencionalidades institucionales que proyectan la investigación desde sus líneas de investigación o desde unos derroteros, enfoques o temas particulares. Estas intencionalidades investigativas se concretan en un punto de vista, sometido al debate con posturas académicas y científicas cercanas al régimen representacional propuesto para la indagación.

En segundo lugar, que más que una búsqueda de referencias sobre un tema en particular, el estado del arte logra afianzar estos intereses en relación con una problemática particular inscrita en los enfoques, tendencias e incluso paradigmas circunscrita en las temáticas o intereses concretos. En tercer lugar, que el planteamiento de un estado del arte se opone para este caso, a las lógicas de construcción de antecedentes donde impera una lógica del enciclopedismo anclada a las prácticas de indagación del siglo XVIII y que valga decir, ha sido domesticada por el positivismo y proyectada como un referente investigativo en las prácticas investigativas contemporáneas y en los escenarios académicos como las 
universidades. En cuarto lugar, más que buscar afianzar un objeto de estudio dado, lo que plantea la lógica del estado del arte es la construcción del objeto de estudio desde la relación entre el sujeto que investiga y el escenario y campo problemático desde el cuál se aborda la indagación.

\section{Operaciones del estado del arte}

Al partir de unos intereses concretos, el estado del arte buscó afianzar un punto de vista en relación a una problemática en un contexto particular. En este orden de ideas todo objeto de estudio que se pretenda construir hace parte de un régimen representacional o una práctica situada que expone de antemano unos mecanismos argumentativos. Para este caso, se entiende por régimen representacional, la postura de la institución en cuanto a su ubicación epistemológica en el campo del patrimonio gastronómico. Para este caso en particular, el abordaje de la realidad no puede estar asociada a formas determinadas de la cultura, sino que admite un diálogo entre diferentes agentes y agencias en la construcción del patrimonio. Habría que comprender que, desde un enfoque construccionista, la realidad no está dada, sino que obedece a las tensiones e interacciones de diferentes agentes y agencias que participan en el proceso de construcción de la realidad. Desde este paraguas epistemológico, la realidad procede bajo interacciones, relaciones y manifestaciones simbólicas de unas representaciones sobre la realidad, que valga decir, a su vez proceden de otras las representaciones de la realidad que han intentado objetivar el mundo social. En esta medida en el estado del arte se requiere una vigilancia epistemológica mayor frente a estos metalenguajes que tienen de por si elementos conceptuales y metodológicos y unas condiciones de producción particulares. Por tal motivo, en la lógica del estado del arte, se logra mayor disquisición epistemológica bajo un ejercicio de reflexividad.

De acuerdo con Serna (2016) desde una práctica investigativa reflexionada, se pretende superar la distinción entre el objeto existente en la realidad y el objeto como producto de la intelección. Estas relaciones entre lo dado y lo conocido y entre la realidad y la representación, requieren implementar en la práctica una profundización conceptual e incluso una rehistorización conceptual, con el propósito de vislumbrar los diferentes lugares 
de enunciación. En este sentido el estado del arte busca dar cuenta de cómo unos autores desde unos campos especializados abordan una problemática investigativa similares al objeto de estudio que se pretende construir. Estas operaciones según Serna (2016) se realizan bajo el presupuesto que dichos autores no investigan en limpio, sino que interponen unos diseños de investigación que, si bien involucran, preguntas, objetivos e hipótesis, también ponen en discusión la pertinencia de unos sistemas teóricos y metodológicos. Es en la profundización conceptual donde es procedente definir tendencias, enfoques e incluso paradigmas.

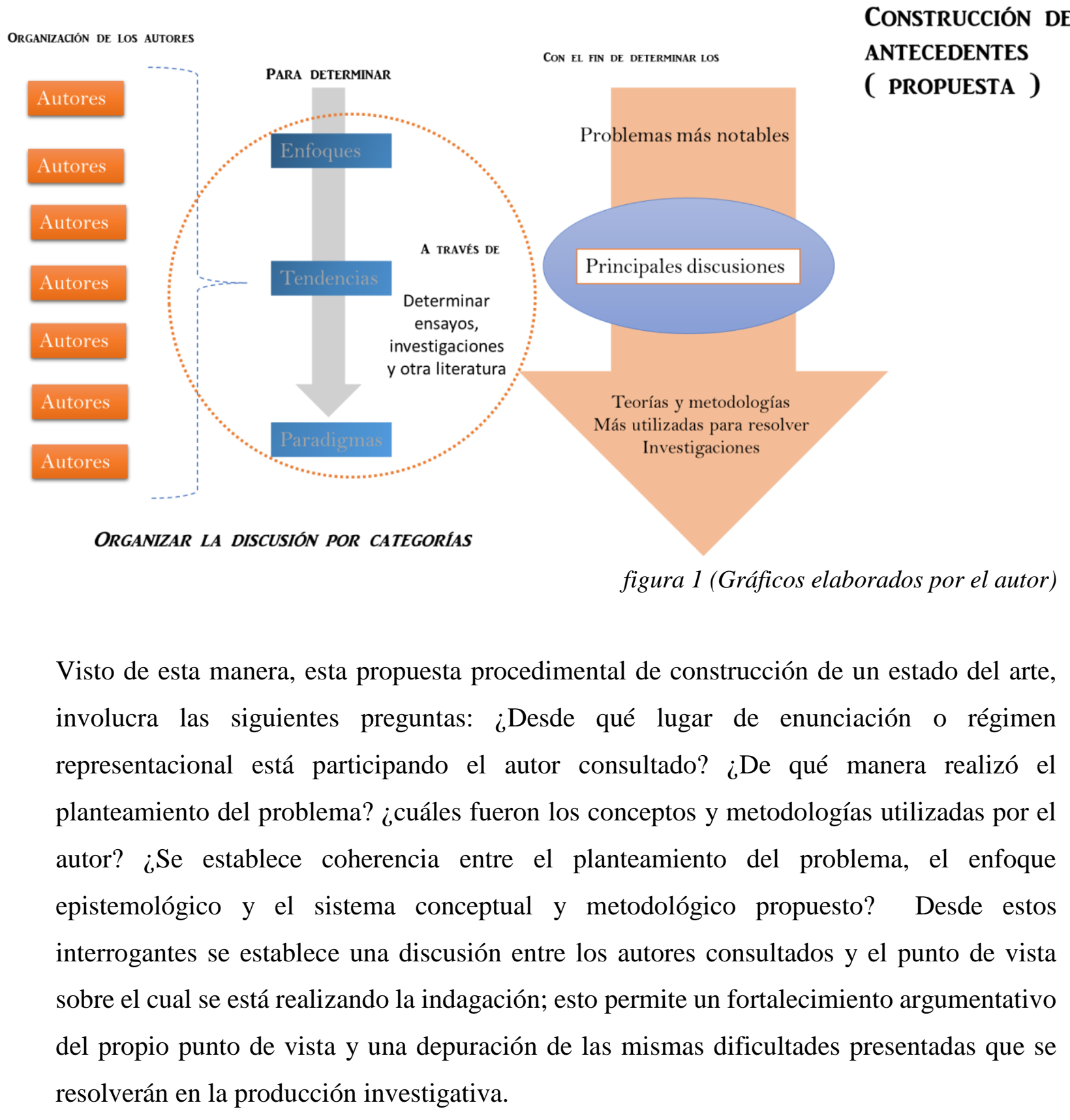




\section{Organización conceptual}

En estos términos un estado del arte procede no solo como el mecanismo para la construcción del objeto de estudio sino además conduce a la proyección investigativa de un punto de vista objetivado que pone en juego la argumentación desde sus fortalezas y debilidades de una práctica situada o un régimen representacional sometido a critica. Desde los planteamientos de Serna (2016) el abordaje del estado del arte procede bajo la construcción de ejes conceptuales que, si bien son provisionales, pueden circunscribir la discusión en torno a una problemática, un contexto y un punto de vista. De este modo, se pasó a definir los ejes conceptuales teniendo en cuenta los intereses institucionales que vinculan el tema gastronómico con los procesos de construcción del patrimonio cultural; aquí vale la pena anotar, que este procedimiento acató las recomendaciones que desde la dirección de investigaciones se realizaron para la elaboración de un estado del arte.

\section{Área de investigación: "Patrimonio y cultura Gastronómica"}

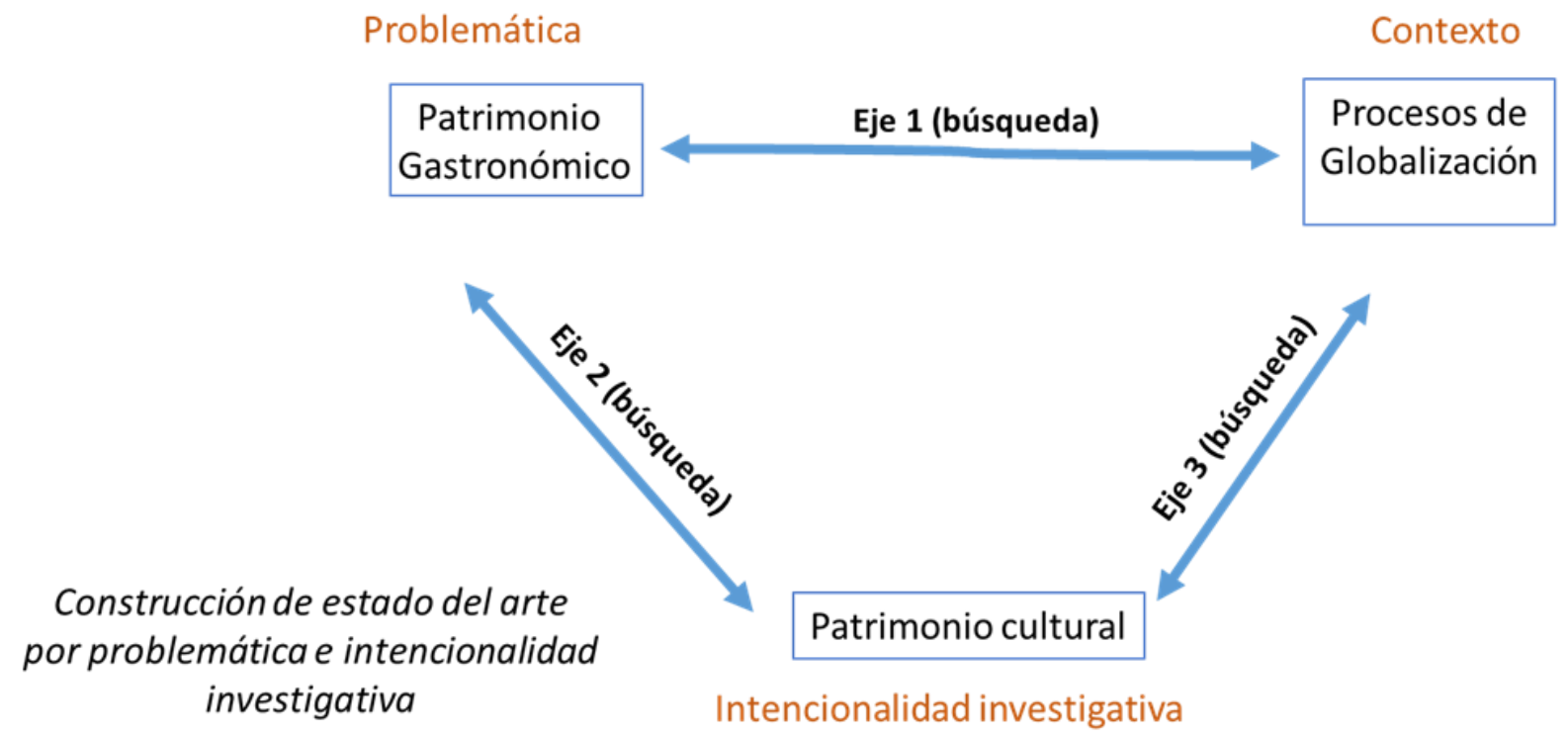

Elaboración propia 
Finalmente, este ejercicio tal como se advierte en el documento orientaciones estados del arte de la Vicerrectoría de Investigaciones, requirió ser sistematizado mediante una matriz Excel (documento adjunto). Cabe mencionar que esta matriz fue construida en un lenguaje sencillo con el propósito de lograr que las fuentes tengan un carácter práctico y facilitar la búsqueda de información para posteriores indagaciones.

\section{Resultados:}

\section{Las tendencias y los enfoques del patrimonio gastronómico como objeto de estudio}

Los resultados obtenidos de esta indagación sobre las diferentes formas de abordaje de un objeto de estudio como el patrimonio gastronómico, permitió entrever diferentes rutas y campos desde los cuales se puede seguir desarrollando investigaciones y líneas de trabajo. En este orden de ideas, los diferentes objetos de estudio y problemas de investigación que se derivan de esta indagación, además alientan reflexiones y tomas de posición frente al futuro inmediato y las formas futuras de abordaje de la investigación en el grupo de investigación denominado Grupo integrado de investigación en Ciencias Culinarias y gastronómicas.

Desde la forma de construcción del estado del arte propuesto en el presente documento es necesario recordar que la búsqueda se realizó por ejes conceptuales. Vale la pena anotar que dentro de la matriz propuesta se incluyeron como eje 1 (la relación entre el patrimonio gastronómico y los procesos de globalización), eje 2 (la relación entre el patrimonio cultural y el patrimonio gastronómico) y el eje 3 (la relación entre los procesos de globalización y el patrimonio cultural). Además, se incluyó otra casilla concerniente a la base de datos con el propósito de analizar la producción académica sobre el tema de lo gastronómico. No obstante, el principal objetivo que cumplió la sistematización y el rastreo bibliográfico fue la de ubicar las tendencias, los enfoques y los paradigmas más importantes en la producción académica sobre el patrimonio gastronómico. En este orden de ideas, los principales resultados fueron los siguientes:

En primer lugar, los estudios que se relacionan en la matriz obedecen a una gran tendencia que abarca la incidencia de la antropología en los estudios sobre la relación entre 
patrimonio gastronómico, patrimonio cultural y procesos de globalización. Esta posición dominante de la antropología en los estudios sobre el acto de cocinar y el sentido humano y biológico de la alimentación, no solo indica un predominio de la antropología de la alimentación en el estudio del patrimonio gastronómico como objeto de estudio, sino que las voces legitimadoras de estos discursos sobre la cocina, las técnicas y los productos de la tierra, con frecuencia apelan directamente a los conceptos antropológicos para explicar las dinámicas del alimento(Gracia Arnaiz M, 2010). Esto no resulta una sorpresa en la medida en que las operaciones intelectuales de estos estudios involucran categorías como prácticas alimentarias, cultura alimentaria, procesos de aculturación, procesos de transculturización de los alimentos y por último procesos de homogeneización cultural bajo los procesos de globalización.

Si bien es cierto que la antropología poco a poco ha cedido su dominio sobre este objeto de estudio a otros regímenes representacionales como las explicaciones semióticas, sociológicas y ahora último desde unas explicaciones económicas e históricas sobre las prácticas alimentarias, esta apertura no ha quebrado por completo el predominio de la antropología en la legitimación de estos discursos. Desde el empoderamiento de obras antropológicas como bueno para comer de Marvin Harris (1996), y mitologías de Levis Strauss, se abrieron campo otros estudios sobre todo de talante sociológico que permitieron una mayor apertura de este campo de estudio como por ejemplo la obra de Goody, Jack (1995) "Cocina, cuisine y clase". Estudio de sociología comparada. De acuerdo con Llano (2015) estos predominios conceptuales han permitido afianzar desde una mirada occidental el discurso de lo gastronómico propio de la cultura francesa, frente a concepciones más locales y populares como la cocina o la comida. Vale la pena decir, que este afianzamiento cultural se ha realizado por medio de la vieja matriz cultural que opone civilización a barbarie, que para este caso pone de manifiesto la tensión entre lo gastronómico y la cocina, siendo esta última práctica un aspecto degradante y excluido del ámbito legitimado del discurso y las prácticas refinadas de lo gastronómico bajo una antigua oposición entre el gastrónomo y el cocinero. 
En segundo lugar, en el estado del arte realizado se logró evidenciar tres tendencias claras en estos estudios. La primera tendencia agrupa los estudios concernientes a dar cuenta de la relación entre los procesos globalizantes y el tema de la identidad cultural. En segundo lugar, la tendencia que agrupa los estudios sobre el patrimonio gastronómico, la identidad cultural y el desarrollo económico territorial y la tercera tendencia, la que acoge los estudios sobre identidad y patrimonio, con un claro sesgo hacia los estudios sobre la relación gastronomía turismo.

En esta primera tendencia fue posible evidenciar que estas discusiones sobre el abordaje del patrimonio gastronómico, se orientan hacia las representaciones y asociaciones del acto alimentario con las dinámicas globalizantes y la cultura de la alimentación. Esta tendencia resulta ser la más dominante de todas, en la medida en que en estos estudios se hace visible una realidad más contemporánea frente al fenómeno gastronómico como el tema cada vez más predominante de las mezclas culturales y el papel que ha cumplido la hiperindustrialización en cuanto a la descontextualización de la cocina y la cultura alimentaria (Lipoveski, 2011).

Al ser cada vez más evidente la industrialización de productos que otrora se ofrecían como productos directos de las costumbres y las tradiciones bajo el marco de la comida tradicional, aparece una crítica cada vez más fuerte frente a la legitimidad de estos productos. Esta crítica obedece a que la producción industrial de la comida, se presenta al consumidor como alimento desprovisto de sus condiciones de producción. Esta operación de marketing permite deslocalizar el producto de sus tradiciones culturales asociadas a la cocina campesina, la cocina popular, e incluso, a las prácticas culturales de la cocina callejera. Otra de las críticas más comunes en esta primera tendencia, se relaciona con el tema de la soberanía alimentaria, que ha sido afectada por los cambios económicos, sociales y culturales que han entrado a influenciar productos nacionales de una tradicional importancia alimentaria (Menasche, R. (s.f).

Estas dinámicas de la globalización económica, cultural, social y tecnológica han afectado en los últimos años la regularidad de la cultura alimentaria local, trastocando los 
estilos de vida, las costumbres y los gustos de los consumidores (Turmo, 2010, Expeixt, 2011; Llano, 2013, Aragón Cánovas F. 2014, Bertrán 2017). En efecto, la importación de nuevos productos y la tendencia a la mezcla cultural y gastronómica sobre las cocinas regionales han resquebrajado modelos conservadores de la cultura para proyectarlos en dinámicas que implican una relación sociedad- mundo bajo un simulacro cultural que por lo general no corresponden con las prácticas reales de los territorios donde se presenta la producción de alimentos (Magaña Carrillo M., Padín Fabeiro C. 2008).

Una segunda postura se centra en la posibilidad de reivindicar a la cocina como un patrimonio mixto que asume las tipificaciones de un elemento cultural tangible e intangible. Desde esta perspectiva los hábitos alimenticios, los alimentos ecológicos y los productos de la tierra se relacionan con el tema identitario (Nunes S. C. 2007) De esta manera, el vínculo entre el producto y el territorio se hace evidente en la visibilidad que puede aportar el turismo alimentario (Ernesto, B. 2013). En este sentido, de acuerdo con Llano (2013) la gastronomía se posicionaría como un elemento fundamental para acceder a culturas desconocidas, convirtiéndose en potenciador del turismo, generando de este modo, nuevas sensaciones por medio de sabores, texturas, colores y formas que podrían convertirse en elementos de reconocimiento al penetrar en las dinámicas locales de producción gastronómica.

Finalmente, una tercera tendencia en este estado del arte permite sostener desde las investigaciones revisadas que existe una propensión del patrimonio gastronómico y la identidad cultural a vincularse cada vez más con el desarrollo territorial (Uribe D, 2006; Fonte M. y Ranaboldo C., 2007, Mascarenhas R. G. y Gândara J. M. 2010, Matta, R. 2011, Ramírez Ortega J. I. 2015). Sin embargo, el desarrollo territorial propuesto en estas discusiones obedece mucho más al rescate de las particularidades de los territorios y sus calidades sensibles para lograr un vínculo del territorio con sus pobladores. Aquí la posibilidad de las cocinas, las técnicas y las tradiciones, permite pensar en las diferentes resistencias culturales y la aparición de movimientos sociales en la defensa de lo agrícola y la soberanía alimentaria. (Pilar, G. 2005; Lozano Cabedo,2006, Delgado Cabeza M. 2013, Gaviria Álvarez L y González F. 2015, Thomé-Ortiz, H. 2015). En esta defensa de lo territorial y sus formas de producción aparecen los discursos contra hegemónicos que 
proponen posibilidades de desarrollo económico en el territorio para hacerle frente a la hiperproducción industrial postcapitalista.

\section{Las posibilidades teóricas del patrimonio gastronómico como objeto de estudio}

Esta revisión de los estudios relacionados con el patrimonio gastronómico deja un panorama conceptual robusto por el lado de las epistemologías de las ciencias sociales. Es importante advertir que la elaboración de un estado del arte desde un régimen representacional basado en el patrimonio cultural como punto de partida estratégico del grupo, obedeció precisamente a sopesar las carencias en el abordaje de lo gastronómico presentes en el mismo grupo. Este abordaje estratégico permitirá a futuro construir puentes entre los estudios relacionados con las prácticas alimentarias, la cultura alimentaria, la historia de la alimentación y en especial en las relaciones del objeto de estudio con las disciplinas de las ciencias sociales. Desde esta puesta en valor se podrán mostrar las propiedades de estos productos desde la innovación tecnológica y el análisis rigurosamente científico del laboratorio.

Cabe anotar que, en la revisión realizada, el saber gastronómico aparece referenciado desde un referente antropológico al ser desplegado como insumo de la cultura. La recomendación en cuanto a esta colonización de este saber por parte de un régimen representacional tan fuerte como la antropología, es precisamente delimitar problemas concretos del saber culinario e intentar unos diálogos con la cultura para complementar la discusión con otras disciplinas como la sociología, la historia, la economía y el turismo bajo un trabajo interdisciplinario.

Este trabajo interdisciplinario incluye un diálogo más abierto y comprometido con las epistemologías de las ciencias naturales. Este intercambio permitirá equilibrar y asumir las diferencias de abordaje teórico, la reflexión epistemológica y el carácter cerrado del método científico. Si se tiene en cuenta que el patrimonio gastronómico es una construcción cultural, entonces el referente y sobre todo la reflexión epistemológica permitirá tejer puentes entre las diferentes escalas de análisis del objeto de estudio. Con el compromiso ético y político de salvaguardar el patrimonio gastronómico, la reflexión epistemológica y teórica tendrá que 
tener mucho cuidado de arrancar los productos del contexto para pasarlos por análisis de laboratorio y producción industrial.

\section{Las posibilidades metodológicas}

Aunque en el estado del arte realizado se privilegia el estudio de caso y las metodologías ligadas a este gran referente disciplinar como la etnografía, el trabajo de campo, la entrevista en profundidad, es importante considerar las posibilidades del trabajo interdisciplinar ${ }^{4}$. En este orden de ideas, las posibilidades teóricas para abordar un objeto de estudio como el patrimonio gastronómico requerirá un trabajo interdisciplinar que logre potenciar por medio de un objeto compartido, el trabajo colaborativo y complementario entre estos dos regímenes representacionales aparentemente opuestos por la dicotomía objetivo subjetivo y las falsas distinciones entre las ciencias duras y las ciencias blandas.

Superada estas falsas fronteras para fortalecer la reflexión interdisciplinaria, se propone entonces un trabajo complementario, que permita, por un lado, dar sentido y poner en valor al patrimonio gastronómico con el trabajo de indagación desde las ciencias sociales y de otro lado, investigar y sistematizar las propiedades de los productos analizados para qué en una tercera operación se ponga en valor el potencial del producto local en la práctica gastronómica. Desde esta última operación relacionada mucho más con el trabajo propiamente curricular y formativo del estudiante de gastronomía, se buscará fortalecer la pertinencia social y académica de unos propósitos de formación y unos perfiles profesionales y ocupaciones de un gastrónomo o cocinero, formado sobre la base del reconocimiento de la producción local y el emprendimiento cultural bajo la difusión de la gastronomía colombiana.

\footnotetext{
${ }^{4}$ Los diferentes estudios de caso de la presente revisión pueden ser explorados en el caso la papa chilota (Santana, R, 1998), el caso peruano en el estudio de Matta, R. (2011); un caso mucho más detallado se puede observar en González Mendoza. M., (2013). Análisis de patrimonio gastronómico entre los mazahuas de San Antonio Pueblo Nuevo, San José del Rincón, México. Y el caso venezolano ver Alcántara M. C., Longa Faría O., Rivas Alfonso B. (2004). La patrimonialización de la gastronomía venezolana como estrategia de desarrollo turístico. Anales Venezolanos de Nutrición, v.17 n.1, N.d. Disponible en: http://www.scielo.org.ve/scielo.php?script=sci_arttext\&pid=S0798-07522004000100004
} 
Finalmente, desde un proceso de investigación que asuma como inicio la contextualización del producto, el análisis de sus propiedades y por último la creación e innovación gastronómica, ya sea desde la reproducción o la transformación del plato local bajo el reconocimiento de su valor social y cultural, se permitirá aterrizar estas conceptualizaciones a las prácticas pedagógicas y didácticas en la formación de un gastrónomo comprometido con la cultura gastronómica de su país.

\section{Conclusiones:}

Los tránsitos culturales del patrimonio gastronómico, de objeto de estudio a objeto de enseñanza

El siguiente documento pretendió ahondar en la discusión sobre los bienes patrimoniales al tener en cuenta que una relación entre patrimonio y gastronomía puede ser además de interesante muy conveniente en la medida en que los diferentes paisajes productivos, alimentos, técnicas, tradiciones se encuentran en un momento clave de reconocimiento (Llano, 2017; Medina, 2017). Para el caso colombiano estas producciones agrícolas y culturales de las diferentes regiones del país están aún por descubrirse. Desde la mezcla cultural y la reivindicación de una nación mestiza, el potencial gastronómico se reconocerá por las influencias de diferentes países europeos, del medio oriente y asiáticos, que han permitido estructurar diferentes tradiciones dispuestas a ser narradas y comunicadas al mundo. Esta representación de una identidad gastronómica asumida desde la mezcla cultural permite pensar en nuestros propios productos de la tierra como se conocen actualmente a los productos agrícolas tradiciones de una región que tienen algunas características que los postulan como productos de alta calidad y de un reconocimiento importante . $^{5}$

Frente a este dilema entre reconocer los procesos culturales y el desarrollo científico, el saber sobre la cocina colombiana requerirá entonces de procesos de investigación

\footnotetext{
${ }^{5} \mathrm{La}$ fama de estos productos se construye por medio de unas condiciones especiales a saber: las técnicas de cultivo que por lo general tiende a lo artesanal junto con unas condiciones geográficas, climáticas y de terreno, entre otros aspectos de su geografía física, muestran que no existe otro producto en otras regiones que se iguale en calidad, en frescura y en autenticidad. Estas propiedades organolépticas de los diferentes productos de la tierra abren la discusión sobre qué es lo que determina su autenticidad, en este sentido aparecen algunos casos que demuestran la complejidad para trazar esta línea divisoria entre lo que es auténtico y lo que se configura como un elemento que copia y roba tradiciones (Llano, 2013).
} 
interdisciplinaria que permita un dialogo abierto en torno a un objeto de estudio como el patrimonio gastronómico. En este sentido, los programas de formación de este talento humano tendrán que tener presente que, bajo el desarrollo investigativo que tiene como propósito el rescate de tradiciones y relatos culinarios no podrá convertirse en un programa de pregrado con una clara vocación sociológica y antropológica, ni tampoco se podrá convertir en un programa de ingeniería de alimentos. La cocina, la comida, las preparaciones, el desarrollo económico y social, tendrán que convertirse en asuntos de reflexión propios de un profesional con clara vocación por la difusión de los platos locales, de los alimentos y sobre todo en un intérprete cultural de las propias preparaciones que hacen parte de un acervo cultural importante asociado a la identidad culinaria del país. Se sugiere desde esta revisión documental que las investigaciones emprendidas por el programa tengan un carácter gastronómico como base y centro de la discusión.

Aunque cabe advertir la presencia de disciplinas como la antropología, la sociología, la economía y el turismo en trabajos sobre cultura alimentaria y patrimonio gastronómico, es importante advertir que quienes requieren liderar los procesos de reconocimiento de la cocina en el país, son precisamente los cocineros. En estos términos el grupo de investigación y los investigadores adscritos a la línea, requieren además de involucrar nuevos perfiles al diálogo de saberes, concretar procesos formativos en cuanto a nuevas formas de abordaje epistemológico y metodológico de unos objetos de estudio que están aún por construirse.

En este orden de ideas una de las recomendaciones para asumir este tipo de investigaciones procede bajo una decisión de perfiles investigativos en el grupo de investigación para buscar un equilibrio entre las discusiones entre las ciencias sociales y naturales, que beneficie directamente a docentes investigadores en formación (profesionales de gastronomía) y a estudiantes en formación. En la medida en que los perfiles están centrados sobre todo en el desarrollo y la innovación de los productos de la tierra asociados a la ingeniera de alimentos, el sentido y la contextualización de los productos y los adelantos para las denominaciones de origen y la puesta en valor del patrimonio pierde capacidad de argumentación por carecer de perfiles asociados a las ciencias sociales y humanas. 
Para lograr comprender esta contextualización e incluso historización de estas formas de estructuración del conocimiento sobre lo gastronómico, se requiere adelantar estudios sociales y culturales que respalden el desarrollo y la innovación. En efecto, como el grupo está asociado a un área estratégica entroncada con el patrimonio cultural y gastronómico, se sugiere la incorporación de perfiles que puedan soportar estas argumentaciones.

Finalmente sin entrar a debatir por la legitimidad de una postura frente a otra, se invita al lector ya sea que se reclame investigador o docente interesado en el tema, a considerar la posibilidad de intercambio y trabajo colaborativo entre estos dos regímenes representacionales que permiten por un lado aportar sentido, reflexión histórica y valor cultural al alimento bajo las miradas de las ciencias sociales y de otro lado, potenciar el alimento bajo posibilidades concretas de desarrollo tecnológico y científico de algunos productos con la mirada de las ciencias naturales.

\section{Referencias}

Alcántara M. C., Longa Faría O., Rivas Alfonso B. (2004). La patrimonialización de la gastronomía venezolana como estrategia de desarrollo turístico. Anales Venezolanos de Nutrición, v.17 n.1, N.d. Disponible http://www.scielo.org.ve/scielo.php?script=sci_arttext\&pid=S0798-07522004000100004.

Aragón Cánovas F. (2014). Gastronomía Y Calidad En Una Europa Globalizada. Revista de Derecho de la Unión Europea, N.4, 1-32. Obtenido de: http://revistas.uned.es/index.php/REDUE/article/view/12668/11864.

Clemente E., Hernández J. M., López-Guzmán T. (2014). La gastronomía como patrimonio cultural y motor del desarrollo turístico. Un análisis DAFO para Extremadura (No. Extra 9th ed.).

Delgado Cabeza M. (2013). El Sistema Agroalimentario Globalizado: Imperios Alimentarios Y Degradación Social Y Ecológica. Revista de Economía Crítica, Pág. 32 - 61. Obtenido de: http://revistaeconomiacritica.org/sites/default/files/revistas/n10/3.pdf.

Ernesto, B. (2013). Las Condiciones De Éxito En La Construcción De Las Rutas Alimentarias. REDMARKA. Revista Digital de Marketing Aplicado., Vol. 1(N.6), Pág. 21 - 49. Recuperado de http://cienciared.com.ar/ra/usr/39/1468/redmarka_n10_pp21_49.pdf. 
Espeitx, E. (2004). Patrimonio alimentario y turismo: una relación singular. PASOS. Revista de Turismo y Patrimonio Cultural, Vol. $2 \mathrm{~N}^{\circ}$ 2, págs. 193-213. Obtenido de: http://pasosonline.org/Publicados/2204/PS040204.pdf.

Fernández G., Agrelo E. (2003). La cultura gastronómica: Un factor clave en el diseño de estrategias de imagen urbana. El Caso de la ciudad de Bernal. Obtenido de: http://hm.unq.edu.ar/hm_publicaciones.html

Fonte M. y Ranaboldo C., (2007). Desarrollo rural, territorios e identidades culturales. Perspectivas desde américa latina y la unión europea. Revista Opera, Universidad Externado de Colombia, No. 7, Pág. 9 - 31. Obtenido de: http://www.redalyc.org/pdf/675/67500702.pdf.

Fusté-Forné, F. (2016). Los paisajes de la cultura: la gastronomía y el patrimonio culinario. Dixit, vol. 24 (1), 1-24. Obtenido de http://www.scielo.edu.uy/scielo.php?script=sci_arttext\&pid=S0797-36912016000100001.

Gaviria Álvarez L., y González F. (2015). Redes alimentarias como resistencia al modelo de globalización dominante: El caso de Slow Food. Slow Food Sevilla y Sur, No. 3, Pág. 1 - 27. Obtenido de: http://www.slowfoodsevillaysur.es/?p=6684.

González Mendoza. M., (2013). Análisis de patrimonio gastronómico entre los mazahuas de San Antonio Pueblo Nuevo, San José del Rincón, México. RI Universidad Autónoma del Estado de México, Paginas 1-115. Obtenido de: http://ri.uaemex.mx/handle/20.500.11799/13729.

Goody, Jack (1995) "Cocina, cuisine y clase". Estudio de sociología comparada, Barcelona, Editorial Gedisa

Gracia Arnaiz M, (2010). Alimentación y cultura en España: una aproximación desde la antropología social. Physis: Revista de Saúde Coletiva, Vol. 20(2), Pág. 357 - 386. Obtenido de: $\quad$ http://www.scielo.br/scielo.php?pid=S0103$73312010000200003 \&$ script=sci_abstract $\&$ tlng=es.

Langreo N. A. (2005). Inmigración y "glocalización" de la alimentación. Revista Distribución y Consumo, N. 80, 42 - 45.

Lourdes, A. (2006). Los debates internacionales en torno al patrimonio cultural inmaterial. Cuicuilco, vol. 13, núm. 38, pág. 13-27. Obtenido de http://www.redalyc.org/pdf/351/35103802.pdf.

Lozano Cabedo, (2006) C Más allá de la patrimonialización de la naturaleza. Alimentos ecológicos y de calidad', en Álvarez, M. y Medina, X. (eds.), Identidades en el plato. Barcelona: Icaria, pp. 63-80. I.S.B.N. 978-84-7426-970-3. 
Llano, Fabián. (2013). Turistas comensales. Patrimonio gastronómico y turismo alimentario en Nemocón y Ubaté. Bogotá: Corporación Unificada Nacional de Educación Superior CUN. Consultado 25 noviembre, 2014. https://www.academia.edu/10681086/Turistas_comensales

Llano, Fabián. (2014). Lo que no se narra se olvida, recuperación de la cocina tradicional en Cundinamarca y Boyacá. Bogotá: Servicio Nacional de Aprendizaje SENA. Consultado 13 enero, 2015. https://www.academia.edu/24307949/Lo_que_no_se_narra_se_olvida

Magaña Carrillo M., Padín Fabeiro C. (2008). ¿Conduce La Globalización A La Pérdida De Costumbres Gastronómicas? Teoría y Praxis, N.05, 213-226. Obtenido de https://reffor.us/index.php.

Mascarenhas R. G. y Gândara J. M. (2010). Producción y transformación territorial La gastronomía como atractivo turístico. Estudios y perspectivas en turismo, vol.19, No. 5. Obtenido de http://www.scielo.org.ar/scielo.php?script=sci_arttext\&pid=S185117322010000500011.

Matta, R. (2011). Posibilidades y límites del desarrollo en el patrimonio inmaterial. El caso de la cocina peruana. En: Apuntes 24 (2): 196-207. Disponible en: http://www.scielo.org.co/pdf/apun/v24n2/v24n2a06.pdf

Medina, X. (2017). Reflexiones sobre el patrimonio y la alimentación desde las perspectivas cultural y turística. Anales de Antropología, 51(2), 106 - 113. Obtenido de: https://www.sciencedirect.com/science/article/pii/S018512251730005X.

Menasche, R. (nd). Cuando La Comida Se Convierte En Patrimonio: Puntualizando La Discusión Disponible en: https://www.academia.edu/19256697/Cuando_la_comida_se_convierte_en_patrimonio_pu ntualizando_la_discusi\%C3\%B3n

MiriamBertran (2017). Domesticar la globalización: alimentación y cultura en la urbanización de una zona rural en México. Anales de Antropología, Vol. 51, Págs. 123-130. Disponible en: https://www.sciencedirect.com/science/article/pii/S0185122517300164.

Nieva, V. L. (2006). Recuperación del patrimonio gastronómico de la provincia de Buenos Aires. Facultad de Ciencias Económicas y Sociales, N. 27, 79 - 93. Disponible en: http://nulan.mdp.edu.ar/169/1/FACES_n27_79-93.pdf.

Nunes S. C. (2007). Somos lo que comemos. Identidad cultural, hábitos alimenticios y turismo. Estudio y perspectiva en turismo, vol. 16, 234 - 242. Obtenido de http://www.scielo.org.ar/pdf/eypt/v16n2/v16n2a06.pdf.

Pérez Sánchez M., Cisneros Mújica W., (2006). El Lado Cultural Del Patrimonio Gastronómico Mexicano. Recuperado junio 2006, de http://web.uaemex.mx/Culinaria/tres_ne/articulo_06.pdf 
Pilar, G. (2005). Resistencia agroecológica a la globalización de la agricultura y la alimentación. El viejo topo, N. 17, 1-3. Obtenido de http://www.rebelion.org/noticia.php?id=10128.

Ramírez Ortega J. I. (2015). Patrimonio Agroalimentario Y Transformaciones Gastronómicas En El Contexto De La Globalización En El Caso De Acambay, Estado De México. RI Universidad Autónoma del Estado de México, Vol.121, 30-33. Obtenido de: http://ri.uaemex.mx/handle/20.500.11799/67878.

Santana, R. (1998). La Papa Chilota Como Patrimonio Cultural. Líder. Vol 5. Obtenido de http://ceder.ulagos.cl/lider/images/numeros/5/1.-

[LIDER\%20VOL5]La\%20papa\%20chilota.pdf

Suremain, C. d. (2017). Cuando la alimentación se hace patrimonio. Rutas gastronómicas, globalización y desarrollo local (México). Rastro. Travaux et Recherches dans les Amériques du Centre, No. 72, Pág. 165-181. Obtenido de http://www.redalyc.org/articulo.oa?id=423853404009.

Thomé-Ortiz, H. (2015). Turismo agroalimentario y nuevos metabolismos sociales de productos locales. Revista Mexicana de Ciencias Agrícolas, vol. 6 (N.6), 30 - 45. Obtenido de http://www.scielo.org.mx/scielo.php?script=sci_arttext\&pid=S200709342015000600018.

Tomás, L., y Margarida, JM (2011). Turismo, Cultura Y Gastronomía. Una Aproximación A Las Rutas Culinarias. Estudios de Turismo y Gestión, VOL. 1, 922 - 929. Obtenido de http://www.redalyc.org/articulo.oa?id=388743867083.

Torres G., Madrid de Zito F. L. y Santoni M. (n.d.). El Alimento, La Cocina Étnica, La Gastronomía Nacional. Elemento Patrimonial Y Un Referente De La Identidad Cultural. Revista Scripta Ethnologica, Vol. XXVI, 55-66. Obtenido de: http://www.redalyc.org/pdf/148/14802603.pdf.Soto

Toselli, C. (2006). Algunas reflexiones sobre el turismo cultural. PASOS. Revista de Turismo y Patrimonio Cultural. , Vol. $4 \mathrm{~N}^{\mathrm{o}}$ 2, págs. 175-182. Obtenido de http://www.pasosonline.org/Publicados/4206/PS040206.pdf.

Turmo, IG (2010). La dieta mediterránea como objeto patrimonial. Quaderns de la Mediterrània, 13, $197 \quad$ - 201. Obtenido de http://www.ethnoterroirs.cnrs.fr/gestion/applis/apetit/fichiers/gonzalesdietemed.pdf.

Uribe D. (2006). La identidad cultural y el desarrollo territorial rural. Una aproximación desde Colombia. Disponible en: http://recursos.salonesvirtuales.com/assets/bloques/Soto_URIBE_desarrolloterritorialrural. $\underline{\mathrm{pdf}}$

Uribe Merino J. F. (2006). Las prácticas alimentarias relacionadas con la búsqueda del ideal corporal. El caso de la ciudad de Medellín (Colombia). Boletín de Antropología; Universidad de Antioquia, No 20(37), Pág. 1 - $24 . \quad$ Obtenido de: http://aprendeenlinea.udea.edu.co/revistas/index.php/boletin/article/view/6897/6314.

Varios autores. (2002). La cocina como patrimonio (in) tangible. Buenos Aires, Argentina: Comisión para la preservación del Patrimonio Histórico Cultural de la Ciudad de Buenos Aires. Disponible en: https://www.folkloretradiciones.com.ar/literatura/temas_6.pdf 
Yudice, G. (2002). El recurso de la cultura. Obtenido de https://www.researchgate.net/profile/George_Yudice/publication/48078079_El_recurso_de _la_cultura/links/0c96051be4c6c0b27a000000/El-recurso-de-la-cultura.pdf 